\title{
Wende, Wolfgang; Walz, Ulrich (Hrsg.) (2017): Die räumliche Wirkung der Landschaftsplanung. Evaluation, Indikatoren und Trends.
}

\author{
Wiesbaden: Springer Spektrum. 198 Seiten, 7 s/w Abbildungen, 32 Farbabbildungen
}

\author{
Stephan Pauleit ${ }^{1}$ (I)
}

Online publiziert: 13. Oktober 2017

(c) Springer-Verlag GmbH Deutschland 2017

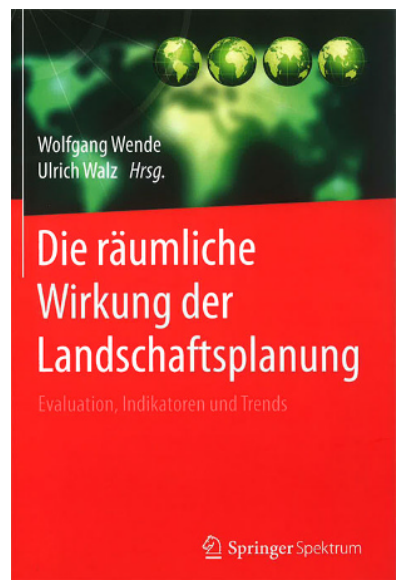

Mit Inkrafttreten des Bundesnaturschutzgesetzes vor 40 Jahren wurde auch die Landschaftsplanung als Instrument eingeführt, um die Ziele des Naturschutzes räumlich zu konkretisieren. Angesichts umfassender und schnell ablaufender Landschaftsveränderungen, ausgelöst etwa durch die Energiewende, die Intensivierung der landwirtschaftlichen Nutzung und Stadtwachstum, erscheint die Landschaftsplanung heute wichtiger denn je, um vorausschauend und ganzheitlich Einfluss auf die Landschaftsentwicklung zu nehmen und nicht nur durch Maßnahmen des Artenschutzes und kompensatorisch auf Eingriffe zu reagieren. Die Landschaftsplanung wurde allerdings nie mit Mitteln, etwa der Bodenordnung oder für investive Maßnahmen, ausgestattet, um selbst ihre Ziele umzusetzen. Nach der anfänglichen

Prof. Dr. Stephan Pauleit

pauleit@tum.de

1 Lehrstuhl für Strategie und Management der Landschaftsentwicklung, Technische Universität München, Emil-Ramann-Straße 6, 85354 Freising, Deutschland
Aufbruchstimmung wurde daher schon bald die Wirkungslosigkeit der Landschaftsplanung bemängelt (Deutscher Bundestag 1987), ohne dies jedoch konkret belegen zu können.

Anlass und Kern des von Wolfgang Wende und Ulrich Walz herausgegebenen Buchs sind die Ergebnisse einer von der Deutschen Forschungsgemeinschaft (DFG) geförderten Untersuchung zu den messbaren Auswirkungen der kommunalen Landschaftsplanung. Das Buch spannt aber in seinen zwölf von führenden deutschen Landschaftsplanerinnen und -planern verfassten Kapiteln einen deutlich weiteren Bogen, beschäftigt sich mit Evaluation, Indikatoren und Trends der Landschaftsplanung.

Im ersten Abschnitt „Evaluation“ zeigen Hartmut Kenneweg und Steffen Tervooren anhand von Fallstudien anschaulich die grundsätzlichen Steuerungsmöglichkeiten der Landschaftsplanung auf - als Fachplanung für den Naturschutz, durch die Integration von Naturschutzzielen in die Bauleitplanung und um Maßstäbe und Prioritäten für die Beurteilung und den Ausgleich von Eingriffen in Natur und Landschaft zu entwickeln. Zudem zeigen sie, wie die Landschaftsplanung durch moderne Geodateninfrastruktur effizienter gestaltet werden kann.

Die folgenden Kapitel im zweiten Abschnitt untersuchen die räumlichen Wirkungen der kommunalen Landschaftsplanung durch Auswertungen von Landschaftsplänen in Deutschland (Christian Stein, Wolfgang Wende und Ulrich Walz) sowie für Thüringen (Dietwald Gruehn). In dem von Christian Stein, Wolfgang Wende und Ulrich Walz vorgestellten DFG-Projekt wurden dazu 600 Gemeinden in Deutschland ausgewählt. Die zum Zeitpunkt der Veröffentlichung noch nicht vollständig ausgewertete Untersuchung ist allein schon aufgrund dieser einzigartigen Empirie wertvoll. Ihre vorläufigen Ergebnisse zeigen, dass das Vorhandensein eines Landschaftsplanes und seine Qualität einen 
statistisch signifikanten Einfluss auf die Landschaftsstruktur und Landschaftsvielfalt haben. Die Landschaftsplanung ist also durchaus wirksam!

Wie kann man überhaupt Landschaftswandel erfassen? Dieser Frage widmen sich im nächsten Abschnitt des Buches drei Kapitel, die unterschiedliche Indikatorensysteme vorstellen. Ulrich Walz und Christian Stein zeigen zunächst Möglichkeiten auf, um im Wesentlichen aus vorhandenen Informationen des Amtlich Topographisch-Kartographischen Informationssystems (ATKIS) Indikatoren zur Flächenneuinanspruchnahme, Zerschneidung durch Infrastrukturen, aber auch zu erneuerbaren Energien und der Veränderung der Landwirtschaft zu gewinnen. Die bundesweite Betrachtung der vorgestellten Indikatoren liefert wertvolle Informationen zu Landschaftsveränderungen, ausgelöst etwa durch landwirtschaftlichen Grünlandumbruch. Dabei werden aber auch die Grenzen des Betrachtungsansatzes diskutiert und Möglichkeiten zur Entwicklung eines umfassenderen Monitorings aufgezeigt. Zudem werden weitere Indikatorensysteme vorgestellt, wie der in der Schweiz entwickelte Ansatz, um die Zersiedelung der Landschaft zu erfassen. Der Ansatz erfasst nicht nur die Veränderung der Siedlungsfläche, sondern unter anderem auch ihre Streuung und Nutzungsdichte. Der Zersiedelungsindikator ist daher sicherlich wesentlich aussagekräftiger als die bloße Flächeninanspruchnahme für Siedlungs- und Infrastrukturzwecke als Messgröße. Die Anwendung dieses Ansatzes in Deutschland stellen Marco Schwarzak und Martin Behnisch vor. Torsten Lipp und Steffen Tervooren zeigen Möglichkeiten für das Landschaftsmonitoring innerhalb von Siedlungen am Beispiel Potsdam auf.

In ,Trends“, dem letzten Abschnitt des Buchse, analysiert Catrin Schmidt die sich beschleunigenden, aber räumlich sehr unterschiedlich ausgeprägten Landschaftsveränderungen durch die Energiewende und die Urbanisierung für Deutschland. Zwischen 2000 und 2030 könnten sich demzufolge etwa zwei Drittel der Landschaftsstrukturen und Landschaftsbilder in Deutschland ganz fundamental ändern. Wie man mit den bereits zur Verfügung stehenden Mitteln der Landschaftsplanung diese Dynamik steuern kann, erläutern sowohl Catrin Schmidt am Beispiel des Kulturlandschaftsprojekts Mittelsachen als auch Markus Reinke und Christina Kühnau anhand eines Fachbeitrags zu erneuerbaren Energien für einen Landschaftsrahmenplan in Bayern. Dass Landschaftsplanung auch als ein sozialer Lernprozess für die daran Beteiligten zu verstehen ist, stellen Christian Albert, Thomas Zimmermann und Ingrid Albert dar. Landschaftsplanung kann demnach besonders zu einer Zunahme von Wissen führen und die Zusammenarbeit zwischen den Planungsbeteiligten fördern.

Ist die Landschaftsplanung also doch erfolgreich? Mit einem „Ja“ beantworten diese Frage Adrian Hoppenstedt und Gottfried Hage, die in der in Deutschland angewand- ten Landschaftsplanung ein auch international zunehmend nachgefragtes Instrument sehen. Sie verweisen aber auch auf Hemmnisse, etwa die wachsende Konkurrenz von Umweltplanungsinstrumenten und die unterschiedlichen Regelungen zur Landschaftsplanung in den einzelnen Bundesländern. Ihre Vorschläge zur Weiterentwicklung der Landschaftsplanung fassen wesentliche Themen der derzeitigen Diskussionen zur Zukunft der Landschaftsplanung zusammen, etwa die Forderung nach einer stärkeren länderübergreifenden Vereinheitlichung von Begriffen, Verfahren und Methoden oder auch der weiteren Modularisierung der Landschaftsplanung, um flexibler auf örtliche Problemlagen reagieren zu können.

Über diese konkreten Erfordernisse an die Weiterentwicklung des Instruments Landschaftsplan hinaus zieht Stefan Heiland in seinem abschließenden Beitrag aus einer breiten Analyse der Stärken und Schwächen der aktuellen Landschaftsplanung Schlussfolgerungen für ihre zukünftige inhaltliche Ausgestaltung. Genannt werden etwa eine stärkere Thematisierung von Landschaftsfunktionen gegenüber der Fokussierung auf die Bewahrung von historisch überkommenen Landschaftsstrukturen und eine stärkere Differenzierung von Zielen und Planungsleitbildern für unterschiedliche Landschaftstypen, die sich nicht an Bilder traditioneller Kulturlandschaften klammern.

Das Buch „Die räumliche Wirkung der Landschaftsplanung “ ist ein wichtiger Beitrag zur Diskussion um die Landschaftsplanung in Deutschland. Es führt den Nachweis, dass Landschaftsplanung tatsächlich steuernden Einfluss auf die Landschaftsentwicklung nehmen kann und genommen hat. Es zeigt aber auch ihre Grenzen auf, etwa bei der Einflussnahme auf die Siedlungsentwicklung und auf die Zerschneidung von Landschaftsräumen. Viele Wirkungen der Landschaftsplanung liegen aber im vorgelagerten Bereich, in dem Einfluss auf andere Planungen und Vorhaben genommen wird. Nicht zuletzt verweist der Beitrag von Christian Albert, Thomas Zimmermann und Ingrid Albert (Kapitel 10) auf die Bedeutung von Landschaftsplanung als kommunikativem Prozess. Dieser Beitrag dürfte für die Diskussion zur Zukunft der Landschaftsplanung von besonderem Interesse sein und wäre zu vertiefen.

Wie sich Landschaftsveränderungen über Indikatorensysteme mit vorhandenen Daten messen lassen, wird im Buch ausführlich dargelegt. Darüber hinaus wäre aber auch die bundesweite Institutionalisierung von Monitoring, das auch die Auswirkungen von Landschaftsänderungen auf Biodiversität, Ökosystemleistungen, Landschaftsbild und Erholungsqualität erfassen sollte, wünschenswert. Zur erfolgreichen Bewältigung der großen Herausforderungen für den Naturschutz, die der schnelle Landschaftswandel mit sich bringt, ist die Weiterentwicklung und Stärkung der Landschaftsplanung auf kommunaler und regionaler Ebene erforderlich. Dazu ist nach meiner Auffassung in diesem 
Zusammenhang eine breitere Diskussion zu führen, die auch informelle Planungsinstrumente und das Instrumentarium anderer Sektoren einbezieht. Wie können Instrumente der ländlichen Entwicklung oder andere Instrumente, die über Fördermittel verfügen, besser mit der Landschaftsplanung verknüpft werden?

\section{Literatur}

Deutscher Bundestag (1987): Unterrichtung durch die Bundesregierung. Umweltgutachten 1987 des Rates von Sachverständigen für Umweltfragen. Bonn. = Drucksache 11/568. 